\title{
ADJOINTS, NUMERICAL RANGES, AND SPECTRA OF OPERATORS ON LOCALLY CONVEX SPACES
}

\author{
BY ROBERT T. MOORE 1
}

Communicated by $\mathrm{H}$. Helson, July 23, 1968

1. Introduction. The results announced here are of two sorts. First. two important tools from operator theory on Hilbert spaces, the notions of states and of numerical ranges of operators, are extended to the setting of operator theory on general locally convex spaces. We employ several ideas developed in the work of Lumer [1] for extending these methods to Banach spaces, combining these with the generalizations of classical Banach space methods to the locally convex setting announced by the author in [3].

Second, several related results are announced concerning the approximation of spectra of operators by their numerical ranges. These results are new in the classical settings of Hilbert and Banach spaces as well as in the more general setting. An interesting motivating example on Hilbert spaces, couched in terms of familiar notions, is discussed separately in $\$ 2$, unencumbered by the technical machinery of later sections.

Full details of proof will appear in two places. A short, less technical discussion of these methods as applied to Hilbert spaces, Banach spaces, $B^{*}$ algebras, and Banach algebras will appear in [6]. The general theory, with concrete applications, will be included in the monograph [5], along with the results announced in [4], which use these methods to provide a unified theory of the generation of semigroups.

2. Approximation of spectra in Hilbert spaces. Let $\mathfrak{S}$ be a complex Hilbert space with a fixed reference inner product $(,)_{0}$. Call another inner product $($,$) equivalent to (,)_{0}$ iff the norms $\|u\|=(u, u)^{1 / 2}$ and $\|u\|_{0}=(u, u)_{0}^{1 / 2}$ induce the same topology on $\mathfrak{S}$; we view inner products as "noncanonical" structures associated with an underlying topology, subject to change when convenient.

Then, for any such inner product $($,$) on \mathfrak{W}$, the numerical range is

$$
W(T,(,))=\{(T u, u) \mid u \in \mathfrak{S}, \text { and }\|u\|=1\} .
$$

It is well known that $W(T,()$,$) is a convex set with compact clo-$ sure containing the spectrum of $T$. In fact, if $K=W(T,(,))^{-}$(the

${ }^{1}$ Research supported in part by NSF GP5585. 
closure of $W)$ and $d_{\lambda}=\operatorname{dist}(\lambda, K)$ for $\lambda \notin K$, then the first-order decay estimate holds:

$$
\left\|(\lambda-T)^{-1}\right\| \leqq d_{\lambda}^{-1}
$$

and this property is known to characterize $K$ [7].

THEOREM 0. Let $U$ be any open set containing the closed convex hull $\operatorname{cocl}(\sigma(T))$ of $\sigma(T)$. Then there exists an inner product $($, ) on $H$ equivalent to $(,)_{0}$ such that

$$
\sigma(T) \subset W(T,(,))-\subset U
$$

Furthermore, if

$$
W_{H}(T)=\bigcap\left\{W(T,(,))^{-} \mid(,) \text {equivalent to }(,)_{0}\right\}
$$

is the Hilbertian numerical range of $T$, then

$$
\operatorname{cocl}(\sigma(T))=W_{H}(T) \text {. }
$$

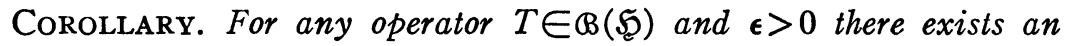
inner product $($,$) such that$

$$
\left\|(\lambda-T)^{-1}\right\| \leqq[\operatorname{dist}(\lambda, \operatorname{cocl}(\sigma(T)))-\epsilon]^{-1} .
$$

3. Geometries, numerical ranges, and first-order decay. The first step in generalizing the theory of $\$ 2$ is to find a suitable geometrical structure on a general locally convex space $\mathfrak{X}$ to replace the inner product on a Hilbert space, and then to extend the numerical range notion. In the language introduced in [3], suppose $\Gamma$ calibrates a complex locally convex space (lcs) $\mathfrak{X}$.

LEMMA 1 (Lumer [1]). Let $p \in \Gamma$. Then there exists a choice function $\chi_{p}: \mathfrak{X} \rightarrow \mathfrak{X}^{*}$ (frequently more than one) such that

$$
\begin{aligned}
& \text { (a) } p(u)^{2}=\left\langle u, \chi_{p}(u)\right\rangle \quad \text { for all } u \in \mathfrak{X} \text {, and } \\
& \text { (b) }\left|\left\langle u, \chi_{p}(v)\right\rangle\right| \leqq p(u) p(v) \quad \text { for all } u \text {, v in } \mathfrak{X} \text {. }
\end{aligned}
$$

(Here $X^{*}$ denotes the space $\mathfrak{L}(\mathfrak{X}, \boldsymbol{C})$ of continuous linear functionals from $\mathfrak{X}$ to $\mathbf{C}$ and $u \rightarrow\left\langle u, u_{*}\right\rangle$ denotes the action of $u_{*} \in \mathfrak{X}^{*}$ on $u \in \mathfrak{X}$.)

Then an important part of the ultimate (Lumer) geometry $\Lambda_{*}$ for $\mathfrak{X}$ is defined by selecting for each $p \in \Gamma$ a $\chi_{p}$ and setting $\Lambda_{0}=\left\{\chi_{p} \mid p \in \Gamma\right\}$. For a general densely defined (dd) $A$ on $\mathfrak{X}$, we define a first part of the numerical range:

(9) $W\left(A, \Lambda_{0}\right)=\left\{\left\langle A u, \chi_{p}(u)\right\rangle \mid u \in D(A), \chi_{p} \in \Lambda_{0}\right.$, and $\left.p(u)=1\right\}$.

In general, $W\left(A, \Lambda_{0}\right)$ does not appear to be big enough to imitate the 
numerical range $W(T,()$,$) in \$ 2$; duality must be brought in more strongly by considering adjoints.

Recall first that if $A$ is any densely defined operator on $\mathfrak{X}$, and $\mathfrak{X}^{*}$ is the space of all continuous linear functionals $u_{*}$ on $\mathfrak{X}$, then the adjoint $A^{*}$ of $A$ is defined, for all $u_{*} \in \mathfrak{X}^{*}$ where $u \rightarrow\left\langle A u, u_{*}\right\rangle$ is continuous on the domain $D(A)$, by $\left\langle A u, u_{*}\right\rangle=\left\langle u, A^{*} u_{*}\right\rangle$. If the strong dual of $\mathfrak{X}$ is denoted by $\mathfrak{X}_{\beta}^{*}$, we have the following analog of a familiar Banach space theorem concerning operator norms of adjoints.

THEOREM 2. For every calibration $\Gamma$ for $\mathfrak{X}$, there exists a natural dual calibration $\Gamma_{*}$ for $\mathfrak{X}_{\beta}^{*}$ such that the adjoint map $T \rightarrow T^{*}$ is an isometric isomorphism of $\mathcal{F}_{\Gamma}(\mathfrak{X})$ into $\mathcal{F}_{\Gamma_{*}}\left(\mathfrak{X}_{\beta}^{*}\right)$. Thus $T \rightarrow T^{*}$ carries $\mathcal{F}(\mathfrak{X})$ into $\mathcal{F}\left(\mathfrak{X}_{\beta}^{*}\right)$.

Picking a $\chi_{q}$ for each $q \in \Gamma_{*}$, we may form a set $\Lambda_{1}=\left\{\chi_{q} \mid q \in \Gamma_{*}\right\}$. Following a suggestion of Phillips [8], let $\mathfrak{X}^{\odot}$ be the closure of $D\left(A^{*}\right)$ in $\mathfrak{X}_{\beta}^{*}$, and let $D\left(A^{\odot}\right)=\left\{u_{*} \in D\left(A^{*}\right) \mid A^{*} U_{*} \in \mathfrak{X}^{\odot}\right\}, A^{\odot}=\left.A^{*}\right|_{D\left(A^{\odot}\right)}$. Then in analogy with (9)

$$
W\left(A^{\odot}, \Lambda_{1}\right)=\left\{\left\langle A^{\odot} u_{*}, \chi_{q}\left(u_{*}\right)\right\rangle \mid u_{*} \in D\left(A^{\odot}\right), \chi_{q} \in \Lambda_{1}, q\left(u_{*}\right)=1\right\} .
$$

Definition 1. Let $\Gamma$ calibrate a complex lcs $\mathfrak{X}$, and let $\Gamma_{*}$ be the dual calibration of $\mathfrak{X}_{\beta}^{*}$.

(a) Then $\Lambda_{*}=\Lambda_{0} \cup \Lambda_{1}$ is a (Lumer) geometry for $(\mathfrak{X}, \Gamma)$.

(b) Furthermore, if $A$ is densely defined on $\mathfrak{X}$

$$
W\left(A, \Lambda_{*}\right)=W\left(A, \Lambda_{0}\right) \cup W\left(A^{\odot}, \Lambda_{1}\right)
$$

is the numerical range of $A$.

Then the following result generalizes $\$ 2$, Lumer [1], and unpublished remarks made to the author by J. Hosack.

Theorem 3. (a) Suppose $\Gamma$ calibrates a complete complex lcs $\mathfrak{X}$ and $A$ is closed dd on $\mathfrak{X}$. Then $W\left(A, \Lambda_{*}\right)-\supset \sigma_{\Gamma}(A)$ and if $d_{\lambda}$ $=\operatorname{dist}\left(\lambda, W\left(A, \Lambda_{*}\right)^{-}\right)$

$$
\left\|(\lambda-A)^{-1}\right\|_{r} \leqq d_{\lambda}^{-1}
$$

for all geometries $\Lambda_{*}$ for $(\mathfrak{X}, \Gamma)$.

(b) Similarly, if $\Gamma$ calibrates a complex normcomplete lcs $\mathfrak{X}$ and $A \in \mathcal{F}_{\Gamma}(\mathfrak{X})$, then the closed convex hull $K=\operatorname{cocl}\left(W\left(A, \Lambda_{0}\right)\right) \supset \sigma_{\Gamma}(A)$ and if $d_{\lambda}=\operatorname{dist}(\lambda, K)$, (12) holds for every $\Lambda_{*}$ for $\Gamma$.

(c) Furthermore, if $K$ is any closed convex set in $C$ such that $K \supset \sigma_{\Gamma}(A)$ and for $d_{\lambda}=\operatorname{dist}(\lambda, K)$ (12) holds, then $K \supset W\left(A, \Lambda_{*}\right)$ for all $\Lambda_{*}$ for $(\mathfrak{X}, \Gamma)$.

(d) In particular, if $\Lambda_{*}$ and $\Lambda_{*}^{\prime}$ are two geometries for $(\mathfrak{X}, \Gamma)$, then in (a) or (b) 


$$
\operatorname{cocl}\left(W\left(A, \Lambda_{*}\right)\right)=\operatorname{cocl}\left(W\left(A, \Lambda_{*}^{\prime}\right)\right) .
$$

4. Convex approximation of spectra. The generalization of Theorem 0 to the locally convex setting seems to require restriction to finite operators $T \in \mathscr{F}(\mathfrak{X})=\bigcup\left\{F_{\Gamma}(\mathfrak{X}) \mid \Gamma\right.$ calibrates $\left.\mathfrak{X}\right\}$; these operators are the ones which most closely imitate "bounded" operators on Banach and Hilbert spaces.

THEOREM 4. Suppose $\mathfrak{X}$ is a complex normcomplete lcs. Let $T \in \mathcal{F}(\mathfrak{X})$ and suppose $U$ is an open set containing the closed convex hull of the finite spectrum $\operatorname{cocl}\left(\sigma_{F}^{*}(T)\right)$. Then there exists a calibration $\Gamma$ for $\mathfrak{X}$ such that if $\Lambda_{*}$ is any geometry for $(\mathfrak{X}, \Gamma)$

$$
\sigma_{F}^{*}(T) \subset W\left(T, \Lambda_{*}\right)^{-} \subset U .
$$

Consequently, in particular

$$
\begin{aligned}
\sigma_{F}^{*}(T) \subset W_{G}(T) \equiv \bigcap\left\{W\left(T, \Lambda_{*}\right)^{-} \mid T \in \mathfrak{F}_{\Gamma}(\mathfrak{X}),\right. \text { and } \\
\left.\Lambda_{*} \text { a geometry for }(\mathfrak{X}, \Gamma)\right\} \subset \operatorname{cocl}\left(\sigma_{F}^{*}(T)\right) .
\end{aligned}
$$

The proof of this theorem depends upon a simpler fact which is a corollary of Theorem 4 from [3], the recalibration theorem. We write $|W|\left(T, \Lambda_{*}\right)=\sup \left\{|\lambda| \mid \lambda \in W\left(T, \Lambda_{*}\right)\right\}$ for the numerical radius of $T$ with respect to $\Lambda_{*}$.

LEMmA 5. (a) If $T \in \mathcal{F}(\mathfrak{X})$,

$$
r_{E}(T)=\inf \left\{|W|\left(T, \Lambda_{*}\right) \mid T \in \mathcal{F}_{\Gamma}(\mathfrak{X}), \Lambda_{*} \text { a geometry for }(\mathfrak{X}, \Gamma)\right\} .
$$

(b) Indeed, if $\left\{T_{1}, \cdots, T_{k}\right\}$ is a finite commutative family in $\mathcal{F}(\mathfrak{X})$, and $\epsilon>0$, then there exists a calibration $\Gamma$ with $\left\{T_{1} \mid 1 \leqq i \leqq k\right\}$ $\mathcal{F}_{\Gamma}(\mathfrak{X})$ such that for every $\Lambda_{*}$ for $(\mathfrak{X}, \Gamma)$

$$
r_{E}\left(T_{i}\right) \leqq|W|\left(T_{i}, \Lambda_{*}\right) \leqq r_{E}\left(T_{i}\right)+\epsilon, \quad 1 \leqq i \leqq k .
$$

No satisfactory answer is known at present to the question: When is the limit in (16) of Lemma 5(a) achieved for some equivalent geometry $\Lambda_{*}$ ? That is, when do we have

$$
r_{E}(T)=|W|\left(T, \Lambda_{*}\right) ?
$$

If $\Lambda_{*}$ is a geometry for a Banach space $(\mathfrak{X},\|\cdot\|)(\Gamma=\{\|\cdot\|\})$ and $T$ is $\Lambda_{*}$-Hermitian $\left(W\left(T, \Lambda_{*}\right) \subset R\right)$, then Vidav [9] has essentially shown that (18) holds. Also, on a Banach space $(\mathfrak{X},\|\cdot\|),(18)$ will occur if the operator Banach algebra generated by $T$ and $I$ is topologically isomorphic to its Gelfand transform function algebra (with the uniform norm). 
5. Decomposition of numerical ranges by projections. Unlike numerical ranges defined by inner products, general numerical ranges need not be convex; systematic exploitation of this nonconvexity can provide useful tools in operator theory. For example, the first part $W\left(T, \Lambda_{0}\right)$ of the numerical range can exhibit the same sort of decomposition with respect to projections as does the spectrum. Call $\Re=\left\{E_{i} \mid 1 \leqq i \leqq k\right\}$ a (finite) resolution of the identity for $T$ if $\sum E_{i}=I$, $E_{i} E_{j}=\delta_{i j} E_{j}$ and $E_{i} T=T E_{i}$ for all $i, j$. Let $T_{i}$ denote the restriction of $T$ to the closed invariant subspace $\mathfrak{X}_{i}=E_{i} \mathfrak{X}$. It is easy to check that $\sigma_{F}^{*}(T)=\bigcup\left\{\sigma_{F}^{*}\left(T_{i}\right) \mid 1 \leqq i \leqq k\right\}$ as a "canonical" topological fact, but careful choice of $\Gamma$ and $\Lambda_{0}$ leads to the following analog for numerical ranges.

LEMMA 6. In the above situation, suppose $\Lambda_{*}^{i}$ is an equivalent geometry for $\mathfrak{X}_{i}$ (with the relative topology). Then there exists an equivalent geometry $\Lambda_{*}$ for $\mathfrak{X}$ with first part $\Lambda_{0}$ satisfying

(a) every $\chi^{i} \in \Lambda_{0}^{i}$ is the restriction of a $\chi \in \Lambda_{0}$, and

$$
\text { (b) } W\left(T, \Lambda_{0}\right)=U\left\{W\left(T_{i}, \Lambda_{0}^{i}\right) \mid 1 \leqq i \leqq k\right\} \text {. }
$$

REMARK. Even in the simplest cases, one cannot hope for a simultaneous decomposition like the above for the second part $W\left(T, \Lambda_{1}\right)$ as well.

This result and Theorem 3(b) allow one to piece together a better approximation theorem for the spectrum.

Theorem 7. Continuing as above, suppose $U_{i} \supset \sigma^{*}\left(T_{i}\right)$ for $1 \leqq i \leqq k$. Then there exist geometries $\Lambda_{*}^{i}$ for the $\mathfrak{X}_{i}$ and $\Lambda_{*}$ for $\mathfrak{X}$ such that if $Q=U\left\{\operatorname{cocl}\left(W\left(T_{i}, \Lambda_{0}^{i}\right)\right) \mid 1 \leqq i \leqq k\right\}$, then $Q \supset \sigma_{F}^{*}(T)$ and for the associated calibration $\Gamma$

$$
\left\|(\lambda-T)^{-1}\right\|_{\Gamma} \leqq \operatorname{dist}(\lambda, Q)^{-1}
$$

These and similar methods enable one to obtain quite detailed information, of numerical range type, about the spectrum and resolvent decay of operators with ample supplies of spectral projections. Many of the ideas date back to Dunford [2]; connections with the active current literature for locally convex spaces will be discussed in later papers.

6. Perturbation of numerical ranges. For many purposes, the dependence of the numerical range $W\left(T, \Lambda_{*}\right)$ of a continuous $T \in \mathfrak{L}(\mathfrak{X})$ upon $T$ is much more accessible than is the comparable dependence of $\sigma_{F}^{*}(T)$ on $T$. We write $\mathscr{L}_{s}(\mathfrak{X})$ and $\mathfrak{L}_{b}(\mathfrak{X})$ for $\mathfrak{L}(\mathfrak{X})$ with the topology of simple convergence and bounded convergence respectively. 
Proposition 8. Let $\Gamma$ calibrate $a$ lcs $\mathfrak{X}$ and let $\Lambda_{*}$ be a geometry for $(\mathfrak{X}, \Gamma)$. Suppose that for some net $\left\{T_{\alpha} \mid \alpha \in J\right\}$ in $\mathscr{L}(\mathfrak{X})$ and some closed $K \subset C, W\left(T_{\alpha}, \Lambda_{*}\right) \subset K$ for all $\alpha \in J$.

(a) If $T_{\alpha} \rightarrow T$ in $\mathfrak{L}_{b}(\mathfrak{X})$, then $W\left(T_{\alpha}, \Lambda_{*}\right) \subset K$.

(b) If $\mathfrak{X}$ is reflexive, and $T_{\alpha} \rightarrow T$ in $\mathscr{L}_{*}(\mathfrak{X})$, then $W\left(T, \Lambda_{*}\right) \subset K$.

Proposition 9. Suppose $T_{0} \in \mathcal{F}_{\Gamma}(\mathfrak{X})$ and $U$ is an open set containing

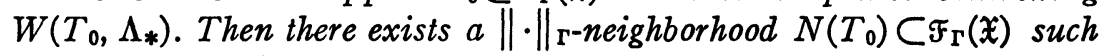
that if $T \in N\left(T_{0}\right), W\left(T, \Lambda_{*}\right) \subset U$.

Proposition 10. Let $\Lambda_{*}$ and $\Gamma$ be as in Proposition 9 and suppose $S, T$ are in $\mathcal{L}(\mathfrak{X})$. Then

$$
W\left(S+T, \Lambda_{*}\right) \subset W\left(S, \Lambda_{*}\right)+W\left(T, \Lambda_{*}\right) .
$$

REMARK. Although the assumptions that $T$ be continuous and that $X$ be reflexive would be unrealistic in many of the classical Banach space formulations of such theories as existence and uniqueness of solutions to integro-differential equations, both assumptions are typically satisfied in the modern locally convex test-function/distribution formulations.

\section{REFERENCES}

1. G. Lumer, Semi-inner product spaces, Trans. Amer. Math. Soc. 100 (1961), $29-43$.

2. N. Dunford, Spectral operators, Pacific J. Math. 4 (1954), 321-364.

3. R. T. Moore, Banach algebras of operators on locally convex spaces, Bull. Amer. Math. Soc. 75 (1969), 68-73.

4. - Generation of equicontinuous semigroups by hermitian and sectorial operators, (in preparation).

5. - Operator theory on locally convex spaces I: Banach algebras, states and numerical ranges (in preparation).

6. - Approximating spectra by numerical ranges (in preparation).

7. G. Orland, On a class of operators, Proc. Amer. Math. Soc. 15 (1964), 75-80.

8. R. S. Phillips, The adjoint semigroup, Pacific J. Math. 5 (1955), 269-283.

9. I. Vidav, Eine metrische Kennzeichnung der selbstadjungierten Operatoren, Math. Z. 66 (1956), 121-128.

University of California, Berkeley, California 94720 